\title{
Monitoria acadêmica na formação em/para a saúde: desafios e possibilidades no âmbito de um currículo interprofissional em saúde
}

\author{
Academic monitoring in health teaching: challenges and possibilities \\ in an interprofessional health curriculum
}

Geovannia Mendonça Santos' ${ }^{1}$, Sylvia Helena Souza da Silva Batista1,2

'Programa de Pós-Graduação Interdisciplinar em Ciências da Saúde, Universidade Federal de São Paulo (UNIFESP) - Santos (SP), Brasil.

²Programa de Pós-Graduação em Psicologia da Educação, Pontifícia Universidade Católica de São Paulo (PUC-SP) - São Paulo (SP), Brasil.

DOI: http://dx.doi.org/10.7322/abcshs.v40i3.796

\section{RESUMO}

Introdução: Este artigo teve origem na pesquisa "Monitoria na universidade: um estudo no âmbito de um currículo interprofissional em saúde - experiências de estudantes do campus Baixada Santista". O referencial teórico foi constituído pelo diálogo com a literatura sobre a monitoria na universidade, bem como com o eixo conceitual de habitus de Pierre Bourdieu. Objetivo: Discutir a monitoria desenvolvida na Universidade Federal de São Paulo (UNIFESP), Campus Baixada Santista, como potencial espaço de formação em/para a saúde a partir das percepções dos discentes sobre a experiência de ser monitor dentro da proposta de um currículo interdisciplinar e interprofissional. Métodos: Os dados foram produzidos a partir da aplicação de questionário a 41 monitores e submetidos à análise de conteúdo do tipo temática. Resultados: A monitoria contribui para o desenvolvimento pessoal do monitor, superando atitudes e posturas frente ao cotidiano acadêmico e ampliando as possibilidades de inserção nas experiências profissionais. Conclusão: $O$ estudante que se predispõe a desempenhar a função de monitor dentro da proposta curricular do Campus Baixada Santista se depara com situações que o preparam para a prática em saúde na perspectiva da interprofissionalidade, da diversidade, do trabalho em equipe e da troca de saberes.

Palavras-chave: monitoramento; acreditação de programas; ensino superior; diretrizes para o planejamento em saúde.

\begin{abstract}
Introduction: This article comes from the research "Academic Monitoring: a study of monitors from Campus Baixada Santista, Universidade Federal de São Paulo". The theoretic framework was made up of the dialogue with the literature of monitoring at the university, as well as the conceptual axis habitus of Pierre Bourdieu. Objective: Discuss the Universidade Federal de São Paulo/Campus Baixada Santista's academic monitoring as a potential health training space from the students perceptions about the experience of being monitor inside an interdisciplinary and interprofessional curriculum. Methods: The data were produced from a questionnaire to 41 monitors and submitted to thematic content analysis. Results: The academic monitoring contributes to the personal development of the monitor, overcoming attitudes and postures and expanding the possibilities of integration in professional experiences. Conclusion: The student who predisposes to development the monitor function acting inside the proposal curriculum of Campus Baixada Santista inevitably comes across situations that prepare them for health practice, from the perspective of interprofessional work, diversity, teamwork and exchange of knowledge.
\end{abstract}

Keywords: monitoring; program accreditation; education, higher; health planning guidelines. 


\section{INTRODUÇÃO}

A monitoria acadêmica pode ser entendida como uma ferramenta de auxílio no processo de ensino-aprendizagem que contribui tanto para o aprendizado do discente quanto do docente ${ }^{1-3}$.

No Brasil, ela foi instituída em 28 de novembro de 1968 pela Lei no 5.540 , que, no artigo 41, fixa normas de funcionamento do ensino superior ${ }^{4}$, sendo revogada em 1996 pela Lei no 9.394 (Lei de Diretrizes e Bases da Educação Nacional).

Por se tratar de uma ferramenta do processo de ensino-aprendizagem que há muito tempo é explorada, assim como outros campos, a monitoria acadêmica deve adaptar-se/ser adaptada às demandas atuais para oferecer aos alunos novas maneiras de apreender os diferentes conteúdos ${ }^{1-3,5}$.

Poucas pesquisas relatam o processo didático do exercício da monitoria acadêmica e o que se observa é que em muitas universidades a monitoria acadêmica ainda é vista com estereótipos que, por vezes, afastam tanto os alunos que necessitam do auxílio da monitoria quanto os alunos que possuem interesse em se tornar monitores ${ }^{1-3}$

As Diretrizes Curriculares Nacionais para os cursos da área da saúde, aprovadas entre 2001 e 2004, apontam para a formação de profissionais de saúde com competências e habilidades para atuar no enfrentamento dos desafios para o desenvolvimento do Sistema Único de Saúde (SUS), considerando a Reforma Sanitária Brasileira e o histórico nacional. A formação, nesse sentido, deve entender a saúde como um processo de construção coletiva, pressupondo a formação de um profissional de saúde crítico-reflexivo.

Para a efetivação dessa proposta de formação, pautada nas ações de prevenção e promoção de saúde e na integralidade do cuidado, faz-se necessária a adequação dos currículos e a diversificação dos cenários de ensino-aprendizagem.

$\mathrm{Na}$ ausência de ferramentas que padronizem e preparem os estudantes a exercerem a monitoria acadêmica, como são desenvolvidas as habilidades que são essenciais para a monitoria no aluno que se propõe a ser monitor?

Em busca de pistas para a construção dessa resposta, emerge a interlocução com Pierre Bourdieu (1930-2002), sociólogo francês que ao longo de suas pesquisas estudou as diferentes relações sociais que se estabelecem e se reproduzem e utilizou o sistema de ensino por ser o ambiente que, de acordo com ele, favorece e permite que tais interações ocorram ${ }^{6}$.

Bourdieu analisa o sistema de dominação que se reproduz no âmbito escolar e a partir disso discute as formas que legitimam essa dominação, bem como o modo como são constituídas as relações sociais dentro desse ambiente.

No livro A reprodução, Bourdieu apresenta o conceito de habitus como um produto da história, um sistema e disposições duradouras e transferíveis, estruturadas e predispostas a funcionar como estruturante que orienta as ações individuais e coletivas trabalhando com as probabilidades e possibilidades de ação. Não se trata de algo consciente e pode ser expresso por meio de várias ações cotidianas (comportamento em geral, modo de se vestir, etc), ou seja, está em toda ação humana ${ }^{6-9}$.

Em essência, o conceito de habitus busca romper com as interpretações deterministas e unidimensionais das práticas. Quer recuperar a noção ativa dos sujeitos como produtos da história de todo campo social e de experiências acumuladas no curso de uma trajetória individual. Os habitus individuais, produtos da socialização, são constituídos em condições sociais específicas, por diferentes sistemas de disposições produzidos em condicionamentos e trajetórias diferentes, em espaços distintos como a família, a escola, o trabalho, os grupos de amigos e/ou a cultura de massa. ${ }^{9}$ (p.65)

Dessa forma, pretende-se compreender o exercício da monitoria acadêmica, reproduzido pelos alunos, a partir do conceito de habitus.

O objetivo do estudo foi apresentar a monitoria desenvolvida na Universidade Federal de São Paulo (UNIFESP), Campus Baixada Santista, como potencial espaço de formação em/para a saúde a partir das percepções dos discentes sobre a experiência de ser monitor dentro da proposta de um currículo interdisciplinar e interprofissional proposto pelo projeto político-pedagógico (PPP) vigente desde a inauguração do campus.

\section{MÉTODOS}

\section{Contexto de pesquisa}

O processo de expansão da UNIFESP teve início efetivo com a criação do Campus Baixada Santista, oficializada em setembro de 2004. Em outubro de 2005, foi assinada a criação definitiva do campus pelo Ministro da Educação e em dezembro do mesmo ano ocorreu o primeiro vestibular para os cinco cursos de graduação em saúde: Fisioterapia, Terapia Ocupacional, Psicologia, Educação Física (ênfase em saúde) e Nutrição. Em 2009, foi implantado o curso de Serviço Social e, em 2012, o Bacharelado Interdisciplinar em Ciência e Tecnologia, com ênfase em Ciências do Mar.

Os cursos voltados para a área da saúde do Campus Baixada Santista estão inseridos em um PPP inovador que possui em sua grade curricular eixos de formação que direcionam o ensino de graduação, sendo eles:

1. Trabalho em Saúde;

2. O Ser Humano em sua dimensão biológica;

3. O Ser Humano e sua Inserção social;

4. Aproximação a uma prática específica em saúde - este último voltado para a formação específica de cada curso.

Cada eixo possui diferentes módulos com propostas singulares de trabalho.

Atualmente, são desenvolvidas no campus monitorias nos eixos comuns e específicos, nos quais os monitores são 
selecionados a partir de entrevistas/provas designadas e elaboradas por eles mesmos.

\section{Sujeitos de pesquisa}

Dos 70 monitores cadastrados no Edital 2011/2012 do Programa de Monitoria do Campus Baixada Santista, 41 responderam ao questionário que buscava apreender concepções dos monitores sobre a prática da monitoria acadêmica.

Para a composição deste recorte, foram selecionadas as respostas de três questões (“O que é monitoria?", "Quais são as tarefas de um monitor?" e "Você recomendaria a experiência de monitoria para outros alunos? Por quê?") com o objetivo de apreender o lugar ocupado pelas diferentes monitorias exercidas na UNIFESP/ Baixada Santista (BS) na vida dos alunos.

O estudo foi submetido à análise e aprovado pelo Comitê de Ética em Pesquisa da UNIFESP. Os procedimentos de coleta de dados contaram com a anuência dos participantes da pesquisa por meio do Termo de Consentimento Livre e Esclarecido.

\section{Análise dos dados}

Realizou-se o caminho interpretativo ${ }^{10}$, no qual o trabalho analítico inclui:

1. pré-análise, na qual são feitas repetidas leituras, obtendo, assim, um contato intenso com o material, criando-se núcleos direcionadores que orientarão a interpretação final da pesquisa;

2. extração de unidades de contexto das falas transcritas e, também, as unidades de registro presentes em cada uma das unidades de contexto;

3. agrupamento/classificação, de acordo com seus significados, em categorias que direcionarão a finalização da análise.

\section{RESULTADOS E DISCUSSÃO}

Para compreender a concepção de monitoria entre os alunos, foi realizada a pergunta: “O que é monitoria?", possibilitando a construção de duas categorias: "Contribuição ao processo Ensino-Aprendizagem" e "Vivência da Rotina Acadêmica/ Professores".

Na categoria "Contribuição ao Processo Ensino-Aprendizagem", a monitoria é vista como ferramenta de auxílio a outros discentes e de aprendizado:

Acima de tudo é responsabilidade, principalmente para com os alunos de graduação. Eles têm no monitor a espera pela resposta correta, ajuda no método de estudo, trabalhos etc. Afinal, monitor também é aluno, também já cobrou os monitores anteriores. (MAnato.7)

Espaço oportuno para erros e acertos, ampliar e consolidar conhecimentos, experiências, vencer dificuldades, lidar com as diferenças. (MSS.4)
Na categoria "Vivências da Rotina Acadêmica/Professores", os monitores relatam a aproximação com a docência de diferentes formas:

A monitoria é um trabalho muito enriquecedor para o aluno, pois ele tem a oportunidade de visualizar o que se passa nos bastidores das aulas. É um trabalho que o aluno não é um mero subordinado do professor, e sim companheiro de trabalho em uma equipe. (MNut.1)

Nesse núcleo é possível observar que a monitoria é vista pelos alunos como um meio pelo qual podem aprofundar o próprio conhecimento e ajudar outros alunos na compreensão do conteúdo apresentado. É também uma atividade que aproxima o monitor da docência, seja pela proximidade com os professores, seja pelo modo de conduzir o trabalho.

\section{Ser monitor: atividades}

A questão "Quais são as tarefas de um monitor?" possibilitou o reconhecimento de três grandes centralidades: "Foco no trabalho com/e para os estudantes (pares)", "Foco no trabalho com o professor" e "Foco na comunidade acadêmica".

A centralidade "Foco no trabalho com/e para os estudantes (pares)" revela uma multiplicidade de papéis desempenhados pelos monitores com relação aos alunos, sendo os principais:

- facilitar a compreensão da matéria por meio de ferramentas;

- otimizar/facilitar a comunicação entre os pares;

- organizar plantões de dúvidas/encontros para o estudo coletivo;

- aprofundar-se em temas que serão importantes para os alunos;

- orientar alunos quanto ao material de estudo a ser utilizado;

- despertar no aluno interesse pelo módulo/eixo.

Nessa centralidade, os monitores expressaram que facilitar a compreensão da matéria por meio de ferramentas diversas envolve: organização de grupos, elaboração de roteiros e simulados, filmes, dinâmicas, desenhos, indicações de textos, atividades práticas e uso de recursos online.

A centralidade "Foco no trabalho com o professor" abrangeu:

- ajudar docentes em suas tarefas específicas;

- ter disponibilidade para atender às demandas de docentes/ discentes;

- participar de reuniões para discutir/organizar/planejar/montar a monitoria e o módulo em questão;

- acompanhar as aulas referentes ao módulo;

- preparação de atividades para os alunos;

- ter disponibilidade para atender às demandas de docentes/ discentes;

- participar de reuniões para discutir/organizar/planejar/montar a monitoria e o módulo em questão;

- acompanhar as aulas referentes aos módulos;

- ajudar docentes em suas tarefas específicas. 
A terceira centralidade, "Promover atividades relacionadas à Monitoria para a comunidade acadêmica", indica que o PPP tem proporcionado mudanças em diferentes âmbitos da universidade, uma vez que, levando em consideração a compreensão de que a "função" do monitor encontra-se diretamente relacionada aos alunos e docentes, a inclusão da comunidade nas atividades faz parte de uma mudança no modo de fazer e exercer a monitoria, ampliando, assim, a visão da mesma.

Produzimos atividades extra-aula que promovem a discussão de conteúdos de interesse dos discentes e pertinentes ao eixo, com o intuito de instigar a reflexão dos graduandos. (MIS.1)

Este núcleo nos permite inferir que há grande diversidade nas atividades e tarefas que são desempenhadas pelos monitores entrevistados, sendo elas direcionadas aos alunos, aos docentes e à comunidade acadêmica de modo geral.

\section{Monitoria: significados}

$\mathrm{Na}$ questão "O que a monitoria significa para você?", emergiram com força as categorias "Aproximação à prática docente", "Crescimento acadêmico, pessoal e profissional", "Contribuição para a formação de pares/convivência com professores e alunos" e "Aprofundamento no eixo/módulo/matéria escolhido".

No âmbito da "Aproximação à prática docente", os monitores ressaltam o aprendizado diante do cotidiano docente, do planejamento de atividades e das dificuldades vivenciadas:

A oportunidade, mesmo que por curto período de tempo, de vivenciar alguns aspectos da docência (bons e ruins), contribuindo, dessa forma, para o crescimento pessoal e profissional do aluno. (MHF.1)

No que se refere ao "Crescimento acadêmico, pessoal e profissional", os monitores destacaram:

Significa o espaço onde há chances de aprendizado, de crescimento profissional e pessoal. Um espaço de troca de experiências e de descobertas, tanto para a prática profissional quanto a prática docente. (MFisio.3)

Uma grande chance para adquirir mais conhecimento, mais responsabilidade e mais respeito para com o próximo. Características essenciais para qualquer profissional. (MMac.4)

Nas categorias "Contribuição para a formação de pares/convivência com professores e alunos" e "Aprofundamento no eixo/ módulo/matéria escolhido", os alunos destacam o aprendizado mútuo e o espaço para aprofundarem temas de seu interesse:

Espaço de aprofundar meu conhecimento sobre uma matéria que me interessei e de contribuir com os estudantes e com o professor ao mesmo tempo em que estes contribuem para o aprofundamento do meu conhecimento. (MSS.1)

Esta fala abaixo sintetiza o significado da monitoria para os entrevistados:

Para mim a monitoria é uma experiência incrível que proporciona ao graduando a aproximação com outros alunos e com os professores, o desenvolvimento de habilidades de comunicação importantíssimas para a prática profissional, a retomada do conhecimento teórico de maneira mais aprofundada e direcionada, e a oportunidade, mesmo que por curto período de tempo, de vivenciar alguns aspectos da docência (bons e ruins), contribuindo, dessa forma, para o crescimento pessoal e profissional do aluno. (MHF.1)

De modo geral, o significado de ser monitor está relacionado ao binômio ensinar-aprender, sendo uma atividade que promove o desenvolvimento de habilidades que extrapolam o âmbito acadêmico.

\section{A monitoria no Campus Baixada Santista: a multiplicidade de habitus}

A partir dos dados produzidos com os monitores foi possível apreender que, apesar de muitos terem consciência de seu papel, suas atividades e da relação com os docentes, outros vivenciam, no mesmo cotidiano, monitorias muito diferentes, podendo esse fato ser justificado pela ausência de um modelo de trabalho a ser desempenhado. Assim, é possível afirmar a existência de uma multiplicidade de monitorias na UNIFESP/BS.

A despeito de compartilharem, monitores e professores, o mesmo espaço universitário orientado por um PPP que tem como centralidade a interdisciplinaridade e o interprofissionalismo, os monitores são "formados" por professores que possuem habitus (e, portanto, modos de ação) que foram constituídos ao longo de suas vivências. Um estudo ${ }^{11}$ indica que permanente deve ser a atenção com os processos pedagógicos presentes na monitoria acadêmica, superando a reprodução de práticas e modelos.

Nesse sentido, pertinentes são as observações ${ }^{3}$ quanto à necessidade de criação de condições favoráveis ao professor, envolvendo valorização da atividade, locais apropriados para a orientação e recursos educacionais que o auxiliem no processo de ensino-aprendizagem.

Uma questão nuclear apreendida neste estudo refere-se ao desenvolvimento pessoal do monitor, superando atitudes e posturas diante do cotidiano acadêmico, ampliando as possibilidades de maior e melhor inserção nas experiências profissionais. Este achado encontra ressonância em outra pesquisa²

É importante considerar as dificuldades encontradas pelos monitores no decurso da monitoria (...). A superação desses obstáculos engrandece os monitores não somente como estudantes, mas também como futuros profissionais. (p.55) 
Em meio a diferentes construções, modos de ação e multiplicidade de habitus, apreendeu-se a importância da monitoria na e para a formação dos universitários, que, ao vivenciarem o ser monitor, a partir das atividades desempenhadas, reconhecem os seus avanços e suas superações, ampliando concepções e práticas.

A monitoria acadêmica, dentro da proposta de formação interdisciplinar e interprofissional da UNIFESP/BS, aproxima o aluno não apenas dos módulos, proporcionando ao aluno monitor a compreensão da docência de forma mais próxima e ativa.

$\mathrm{O}$ aluno que se predispõe a desempenhar a função de monitor nesse cenário inevitavelmente se depara com situações que o preparam para a prática em saúde, como a atuação interdisciplinar, o trabalho em grupo, a resolução de problemas, etc.
Nesse sentido, é possível pensar a monitoria acadêmica, no processo de formação do profissional de saúde, como uma ferramenta que vai ao encontro do que é apontado pelas Diretrizes Curriculares Nacionais para os cursos da área da saúde.

O exercício da monitoria acadêmica e seu reconhecimento no âmbito da formação em/para a saúde amplia os cenários possíveis de aprendizagem, fortalecendo a formação de um perfil de profissionais de saúde que dialogam com as demandas do SUS.

Por se tratar de uma atividade extracurricular que aproxima o aluno da docência, e por esse interesse, na maior parte das vezes, ser estimulado a partir dos modelos vividos, faz-se necessário compreender a concepção de monitoria dos professores que atuam nos diferentes projetos de monitoria, promovendo espaços de formação e diálogo sobre o tema.

\section{REFERÊNCIAS}

1. Assis F, Borsatto AZ, Silva PDD, Peres PL, Rocha PR, Lopes GT. Programa de Monitoria Acadêmica: percepções de monitores e orientadores. Rev Enferm UERJ. 2006;14(3):391-7.

2. Cechinel MP, Lopes GT, Oliveira ACH, Camacho KG. As relações sociais entre os diferentes sujeitos da monitoria acadêmica em um centro biomédico. Rev Enferm UERJ. 2005;13(1):51-6.

3. Natário EG, Santos AAAS. Programa de monitores para o ensino superior. Estud Psicol. 2010;27(3):355-64. http://dx.doi.org/10.1590/S0103-166X2010000300007

4. Brasil. Câmara dos Deputados. Centro de Documentação e Informação. Lei n 5.540, de 28 de novembro de 1968. Fixa normas de organização e funcionamento do ensino superior e sua articulação com a escola média, e dá outras providências. Disponível em: http:// www2.camara.leg.br/legin/fed/lei/1960-1969/lei-5540-28-novembro1968-359201-normaatualizada-pl.pdf. Acesso em: 06 jun. 2015.

5. Sobral DT. Valor e significado da vivência no primeiro ano do curso de medicina: apreciação de aprendizado pessoal e contexto em uma série histórica. Rev Bras Educ Med. 2008;32(1):23-31. http://dx.doi.org/10.1590/S0100-55022008000100004
6. Stival MCEE, Fortunato SAO. Dominação e Reprodução na Escola: Visão de Pierre Bourdieu. Disponível em: http://www.pucpr.br/ eventos/educere/educere2008/anais/pdf/676_924.pdf. Acesso em: 06 jun. 2015.

7. Pies N. A Concepção do habitus em Pierre Bourdieu. Tese (Mestrado em Educação) - Universidade de Passo Fundo, Passo Fundo, 2009

8. Bourdieu P, Passeron JC. A reprodução: elementos para uma teoria do sistema de ensino. Rio de Janeiro: Francisco Alves; 1975

9. Setton MGJ. A teoria do habitus em Pierre Bourdieu: uma leitura contemporânea. Rev Bras Educ. 2002;(20):60-70. http://dx.doi.org/10.1590/S1413-24782002000200005

10. Franco MLPB. Análise de conteúdo. Brasília: Plano; 2003.

11. Borsatto AZ, Silva PDD, Assis F, Oliveira N-ECCO, Rocha PR, Lopes GT. Processo de implantação e consolidação da monitoria acadêmica na UERJ e na Faculdade de Enfermagem (1985-2000). Esc Anna Nery. 2006:10(2):187-94. http://dx.doi.org/10.1590/S1414-81452006000200004 